\title{
Atributos químicos e estoques de carbono em Latossolo sob plantio direto no cerrado do Piauí
}

\author{
Luiz F. C. Leite ${ }^{1}$, Sandra R. S. G alvão ${ }^{1}$, Manoel R. Holanda Neto ${ }^{2}$, \\ Fernando S. Araújo ${ }^{3} \&$ Bruna F. Iwata $^{4}$
}

\begin{abstract}
RESU MO
O sistema de plantio direto é uma alternativa viável para o uso sustentável dos solos. 0 objetivo do presente trabalho foi avaliar a influência do sistema plantio direto com diferentes tempos de implantação e do plantio convencional sobre os atributos químicos e os estoques de $\mathrm{C}$ de um Latossolo VermelhoAmarelo do cerrado piauiense. Amostras de solo foram coletadas nas camadas de 0-5, 5-10, 10-20 e 20$40 \mathrm{~cm}$ em sistema de plantio convencional (PC) e plantio direto (PD) com 2 (PD 2), 4 (PD 4) e 6 (PD 6) anos de implantação, além de uma área sob floresta nativa de cerrado (FNC). Foram determinados o pH em água, $\mathrm{P}$ extraível (Pext), bases trocáveis, $\mathrm{Al}$, acidez potencial $(\mathrm{H}+\mathrm{Al})$, carbono orgânico total (COT) e carbono da biomassa microbiana $\left(C_{\text {mic }}\right)$. A adoção do sistema plantio direto aumentou o $\mathrm{pH}$, os teores de $\mathrm{P}$ e de bases trocáveis do solo, em comparação com o PC. Em relação ao PC, os estoques de CO T e $\mathrm{C}_{\text {mic }}$ aumentaram 34 e $99 \%$ no PD2, 47 e 92\% no PD 4 e 61 e $108 \%$ no PD 6, respectivamente, na camada superficial de $0-20 \mathrm{~cm}$. A implantação do sistema plantio direto melhora os atributos químicos e aumenta os estoques de carbono orgânico total e microbiano do solo e seu uso contínuo contribui para a qualidade do solo em áreas do cerrado piauiense.
\end{abstract}

Palavras-chave: sistemas de preparo do solo, fertilidade do solo, carbono da biomassa microbiana, Glycine max L.

\section{Chemical attributes and carbon stocks in an Oxisol under no-tillage in savannah of Piauí state}

\begin{abstract}
No-tillage adoption has been considered a viable alternative for sustainable soil use. This work aimed to evaluate the influence of no-tillage system with different times of adoption and conventional tillage on chemical attributes and carbon stocks in an 0 xisol of the savannah of Piauí in N ortheastern Brazil. Soil samples in the $0-5,5-10,10-20$ and $20-40 \mathrm{~cm}$ layers were collected in conventional tillage (CT) no-tillage (NT) with 2 (NT2), 4 (NT4) and 6 (NT6) years of adoption and native forest (N F). The following chemical properties were evaluated: $\mathrm{pH}$, extractable $\mathrm{P}$, exchangeable cations, $\mathrm{Al}$, potential acidity $(\mathrm{H}+\mathrm{Al})$, total organic carbon (TOC) and microbial biomass carbon $\left(\mathrm{C}_{\text {mic }}\right.$ ). NT adoption increased $\mathrm{pH}$, extractable $\mathrm{P}$ and exchangeable cations contents. Compared to conventional tillage, TO $\mathrm{C}$ and $\mathrm{C}_{\text {mic }}$ stocks increased 34 and 99\% (NT2), 47 and 92\% (NT4) and 61 and 108\% (NT6), respectively, in the superficial layer (0-20 $\mathrm{cm}$ ). No-tillage adoption improves chemical attributes and increases total organic carbon and microbial carbon and its continuous use contributes to soil quality in savannah soils of Piaui state.
\end{abstract}

Key words: soil tillage system, soil fertility, microbial biomass carbon, Glycine max L.

\footnotetext{
Embrapa Meio-Norte, Av. Duque de Caxias, 5650, Buenos Aires, CEP 64006-220, Teresina, PI. Fone: (86) 3089-9185. E-mail: luizf@cpamn.embrapa.br; reginassg@uol.com.br

2 FAEM/UFPel, Rua Gomes Carneiro, 01, Centro, CEP 96010-610, Pelotas, RS. Fone: (53) 3921-1401. E-mail: mrholandaneto@yahoo.com 3 FEAGRI/U NICAM P, Av. Candido Rondon, 501, Barão Geraldo, CEP 13083-875, Campinas, SP. Fax: (19) 3521-1005.E-mail: agronando16@hotmail.com.

${ }^{4}$ PPGA/U FPI, Campus U niversitário M inistro Petrônio Portella, Bairro Ininga, CEP 64049-550, Teresina, PI. E-mail: iwatameioambiente@gmail.com
} 


\section{INTRODUÇÃO}

O cerrado piauiense ocupa uma área de quase 12 milhões de hectares, o que corresponde a $46 \%$ da área do Estado, 5,9\% do cerrado brasileiro e $36,9 \%$ do cerrado nordestino. Do total, $70 \%$ correspondem à área de domínio e os 30\% restantes compreendem a vegetação de transição com o bioma caatinga (Aguiar \& Monteiro, 2005). Nas últimas três décadas tem-se observado expressivo aumento das atividades agrícolas em áreas do cerrado, com intensa substituição da vegetação nativa por áreas cultivadas, especialmente para produção de alimentos, fibra e energia (Ferreira et al., 2007).

A transformação desses ambientes naturais para sistemas agrícolas tem provocado a degradação do solo em consequência de sua exploração inadequada (Fonseca et al., 2007). Neste sentido, a adoção de sistemas de manejo do solo considerados conservacionistas, como o plantio direto (PD), tem-se apresentado como alternativa viável para assegurar a sustentabilidade do uso agrícola do solo, principalmente nos Latossolos (Silva et al., 2000; Bayer et al., 2004), considerados ácidos, com baixa fertilidade natural e potencial de produção condicionado ao uso de corretivos e fertilizantes (Azevedo et al., 2007); portanto, para a exploração racional do cerrado piauiense, é essencial corrigir a acidez do solo utilizando-se calagem que, conjuntamente com as práticas de manejo do sistema PD, gera uma expectativa de acúmulo de resíduos vegetais e nutrientes, além de diminuição de processos, como erosão e compactação do solo.

O sucesso do PD decorre do acúmulo de palhada, propiciado pelas culturas de cobertura e restos culturais de lavouras comerciais, possibilitando a criação de um ambiente favorável à recuperação e à manutenção da qualidade do solo (Kliemann \& Braz, 2006). Comumente, o PD está associado à rotação de culturas anuais, que propicia mudanças nas propriedades químicas do solo, causadas sobremaneira pelo aumento nos teores de matéria orgânica (Murage et al., 2007) e variações no pH do solo, na capacidade de troca catiônica (Souza \& Alves, 2003), bem como nas bases trocáveis e no $\mathrm{P}$ disponível (Mallarino \& Borges, 2006; Thomas et al., 2007), além da diminuição do alumínio tóxico, em relação ao sistema convencional (Silveira \& Stone, 2001).

$\mathrm{O}$ aumento nos estoques de $\mathrm{C}$ e o potencial para mitigar emissões de $\mathrm{CO}_{2}$, também atribuídos ao sistema PD, têm sido reportados em diversos estudos (Leite et al., 2003; Bayer et al., 2006) e são associados ao incremento no aporte de resíduo e à ausência do preparo do solo, o qual diminui a exposição do C protegido nos agregados à comunidade microbiana, retardando o processo de decomposição (Al-Kaisi \& Yin, 2005). Por outro lado, alguns autores preconizam que o aumento desses estoques é verificado apenas quando se avaliam até $30 \mathrm{~cm}$ de profundidade e que, abaixo disso, os maiores valores estão no sistema convencional (Baker et al., 2007).

Apesar dos diversos estudos na região centro-sul do Brasil, são escassos, no Cerrado do Piauí, trabalhos que visem avaliar o efeito do sistema PD sobre os atributos do solo e estoques de C. O objetivo do presente estudo foi quantificar os efeitos do sistema plantio direto sob diferentes tempos de implantação e do preparo convencional sobre os atributos químicos e estoques de $\mathrm{C}$ de um Latossolo Vermelho Amarelo do cerrado piauiense.

\section{Material e MÉTODOS}

O estudo foi realizado no município de Baixa Grande do Ribeiro ( $07^{\circ} 33^{\prime} 30^{\prime \prime} \mathrm{S}, 45^{\circ} 14^{\prime} 32^{\prime \prime} \mathrm{W}$ ), na região sudoeste do estado do Piauí. O solo da área está classificado como Latossolo Vermelho-Amarelo textura argilo-arenosa (320, 150, 100 e $430 \mathrm{~g} \mathrm{~kg}^{-1}$, de areia grossa, areia fina, silte e argila, respectivamente). As condições climáticas do município de Baixa Grande do Ribeiro (com altitude da sede a $325 \mathrm{~m}$ acima do nível do mar) apresentam temperaturas mínimas de $26{ }^{\circ} \mathrm{C}$ e máximas de $36^{\circ} \mathrm{C}$, com clima quente e semiúmido. A precipitação pluviométrica média anual é definida no Regime Equatorial Continental, com isoietas anuais em torno de 700 a $1.200 \mathrm{~mm}$ e período chuvoso estendendo-se de novembro-dezembro a abril-maio. Os meses de janeiro, fevereiro e março, formam o trimestre mais úmido (CPRM, 2004).

Foram avaliados, em áreas cultivadas, dois sistemas de preparo do solo: convencional (PC), com uso de grade pesada mais duas grades leves, e plantio direto (PD) com dois (PD2), quatro (PD4) e seis (PD6) anos de implantação. Além disso, avaliou-se também uma área sob floresta nativa de cerrado (FNC), adjacente àquelas sob os sistemas de preparo, e utilizada como referência de um estado de equilíbrio. O histórico completo de manejo das áreas sob PC, PD e FNC, está descrito na Tabela 1.

Tabela 1. Histórico de manejo das áreas sob plantio direto e plantio convencional, no cerrado piauiense

\begin{tabular}{|c|c|c|c|c|c|c|c|c|c|c|c|c|c|}
\hline \multirow{2}{*}{$\begin{array}{l}\text { Sistema } \\
\text { de cultivo }\end{array}$} & \multicolumn{13}{|c|}{ Histórico de manejo } \\
\hline & $\begin{array}{l}1993 / \\
1994\end{array}$ & $\begin{array}{l}1994 / \\
1995\end{array}$ & $\begin{array}{l}1995 / \\
1996\end{array}$ & $\begin{array}{l}1996 / \\
1997\end{array}$ & $\begin{array}{l}1997 / \\
1998\end{array}$ & $\begin{array}{l}1998 / \\
1999\end{array}$ & $\begin{array}{l}1999 / \\
2000\end{array}$ & $\begin{array}{l}2000 / \\
2001\end{array}$ & $\begin{array}{l}2001 / \\
2002\end{array}$ & $\begin{array}{l}2002 / \\
2003\end{array}$ & $\begin{array}{l}2003 / \\
2004\end{array}$ & $\begin{array}{l}2004 / \\
2005\end{array}$ & $\begin{array}{l}2005 / \\
2006\end{array}$ \\
\hline PC & $\begin{array}{l}\text { Mata } \\
\text { nativa }\end{array}$ & Arroz & Arroz & Arroz & Soja & Soja & Soja & Arroz & Soja & Soja & Soja & Soja & Soja \\
\hline PD2 & $\begin{array}{l}\text { Mata } \\
\text { nativa }\end{array}$ & $\begin{array}{l}\text { Mata } \\
\text { nativa }\end{array}$ & $\begin{array}{l}\text { Mata } \\
\text { nativa }\end{array}$ & $\begin{array}{l}\text { Mata } \\
\text { nativa }\end{array}$ & $\begin{array}{l}\text { Mata } \\
\text { nativa }\end{array}$ & $\begin{array}{l}\text { Mata } \\
\text { nativa }\end{array}$ & Soja & Soja & Arroz & Soja & $\begin{array}{l}\text { Milheto/ } \\
\text { Milho }\end{array}$ & $\begin{array}{l}\text { Milheto/ } \\
\text { Soja }\end{array}$ & $\begin{array}{l}\text { Milheto/ } \\
\text { Soja }\end{array}$ \\
\hline PD4 & $\begin{array}{l}\text { Mata } \\
\text { nativa }\end{array}$ & Arroz & Arroz & Arroz & Soja & Soja & Arroz & Soja & $\begin{array}{l}\text { Milheto/ } \\
\text { Soja }\end{array}$ & $\begin{array}{c}\text { Milheto/ } \\
\text { Milho }\end{array}$ & $\begin{array}{l}\text { Milheto/ } \\
\text { Soja }\end{array}$ & $\begin{array}{l}\text { Milheto/ } \\
\text { Soja }\end{array}$ & $\begin{array}{c}\text { Milheto/ } \\
\text { Soja }\end{array}$ \\
\hline PD6 & $\begin{array}{l}\text { Mata } \\
\text { nativa }\end{array}$ & Soja & Arroz & Arroz & Soja & Soja & $\begin{array}{l}\text { Milheto/ } \\
\text { Milho }\end{array}$ & $\begin{array}{l}\text { Milheto/ } \\
\text { Soja }\end{array}$ & $\begin{array}{l}\text { Milheto/ } \\
\text { Algodão }\end{array}$ & $\begin{array}{c}\text { Milheto/ } \\
\text { Arroz }\end{array}$ & $\begin{array}{l}\text { Milheto/ } \\
\text { Soja }\end{array}$ & $\begin{array}{l}\text { Milheto/ } \\
\text { Soja }\end{array}$ & $\begin{array}{c}\text { Milheto/ } \\
\text { Soja }\end{array}$ \\
\hline FNC & $\begin{array}{l}\text { Mata } \\
\text { nativa }\end{array}$ & $\begin{array}{l}\text { Mata } \\
\text { nativa }\end{array}$ & $\begin{array}{l}\text { Mata } \\
\text { nativa }\end{array}$ & $\begin{array}{l}\text { Mata } \\
\text { nativa }\end{array}$ & $\begin{array}{l}\text { Mata } \\
\text { nativa }\end{array}$ & $\begin{array}{l}\text { Mata } \\
\text { nativa }\end{array}$ & $\begin{array}{l}\text { Mata } \\
\text { nativa }\end{array}$ & $\begin{array}{l}\text { Mata } \\
\text { nativa }\end{array}$ & $\begin{array}{l}\text { Mata } \\
\text { nativa }\end{array}$ & $\begin{array}{l}\text { Mata } \\
\text { nativa }\end{array}$ & $\begin{array}{l}\text { Mata } \\
\text { nativa }\end{array}$ & $\begin{array}{l}\text { Mata } \\
\text { nativa }\end{array}$ & $\begin{array}{l}\text { Mata } \\
\text { nativa }\end{array}$ \\
\hline
\end{tabular}

PC - Plantio convencional; PD2 - Plantio direto com 2 anos de implantação; PD4 - Plantio direto com 4 anos de implantação; PD6 - Plantio direto com 6 anos de implantação; FNC - Floresta Nativa de Cerrado. 0 sistema adotado em PD2, PD4 e PD6, após a retirada da mata nativa e anteriormente à implantação do plantio direto, era o convencional, com uso de gradagem pesada e leve 
Em todos os sistemas cultivados, houve correção do solo e adubação, de acordo com a necessidade da cultura implantada implicando, portanto, em uma variabilidade nas quantidades de fertilizantes adicionadas ao solo. O calcário e os fertilizantes foram aplicados a lanço, com exceção da fonte fosfatada, aplicada na linha de plantio.

No ano agrícola 2005/2006, por ocasião da colheita da soja, avaliou-se a produtividade de grãos por meio de quatro amostragens por sistema, as quais foram efetuadas em parcela de 7,20 x $10 \mathrm{~m}$ e área útil de $9 \mathrm{~m}^{2}$. Com base nesta informação, se estimaram os aportes médios de $\mathrm{C}$ e $\mathrm{N}$ pela cultura, utilizando-se a seguinte equação: Aporte de C $(\mathrm{N})=\mathrm{P}(100-\mathrm{U}) / \mathrm{IC} \times \mathrm{C}(\mathrm{N}) / 100$, em que $\mathrm{P}=$ Produtividade da cultura $\left(\mathrm{kg} \mathrm{ha}^{-1}\right), \mathrm{U}=$ Umidade no grão $(\%)$, IC = Índice de colheita $(\%)$ - razão entre massa de grãos e massa total da planta e $\mathrm{C}(\mathrm{N})=$ Percentual de carbono (40\%) ou nitrogênio (2\%) no material vegetal (Leite et al., 2007). Para se obter uma avaliação mais detalhada, os valores estimados foram divididos em raiz e parte aérea/raiz assumindo uma relação parte aérea/raiz de 3,5:1, de acordo com Lestari et al. (2006).

A amostragem do solo nos diferentes sistemas de preparo, nas camadas de $0-5,5-10,10-20$ e $20-40 \mathrm{~cm}$, foi efetuada por meio da abertura, aleatoriamente, de mini-perfis com 0,50 m de profundidade, 0,40 m de largura e 0,60 $\mathrm{m}$ de comprimento, em uma parcela de 7,20 x $10 \mathrm{~m}$, com cada miniperfil correspondendo a uma amostra simples. Quatro amostras simples foram coletadas e formaram uma composta, representativa de cada área amostral. Após a coleta, as amostras destinadas à determinação do carbono da biomassa microbiana (Cmic) foram acondicionadas em sacos plásticos com respirador e transportadas em caixas de isopor com gelo até o laboratório, onde foram homogeneizadas e passadas em peneira com malha de $2 \mathrm{~mm}$ e acondicionadas em câmara fria até serem analisadas. Para as determinações químicas, as amostras foram secas ao ar, destorroadas e passadas em peneira com malha de $2 \mathrm{~mm}$.

Determinaram-se $\mathrm{pH}$ em água $(1: 2,5)$ por potenciometria, e acidez trocável $\left(\mathrm{Al}^{+3}\right)$ extraída com $\mathrm{KCl} 1 \mathrm{~mol} \mathrm{~L}^{-1}$ e quantificada por titulometria com hidróxido de sódio $0,025 \mathrm{~mol} \mathrm{~L}^{-1}$, padronizado com biftalato de potássio. $\mathrm{O}$ fósforo e o potássio foram extraídos com Mehlich-1 e determinados por colorimetria e fotometria de chama, respectivamente. Cálcio e magnésio foram extraídos com $\mathrm{KCl} 1 \mathrm{~mol} \mathrm{~L}^{-1}$ e determinados por espectrofotometria de absorção atômica (EMBRAPA, 1997).

Para determinação dos teores de carbono orgânico total (COT), as amostras de solo foram trituradas em almofariz, passadas em peneira de malha $0,21 \mathrm{~mm}$ e quantificadas por oxidação da matéria orgânica via úmida, empregando-se solução de dicromato de potássio em meio ácido, com fonte externa de calor (Yeomans \& Bremner, 1988). A biomassa microbiana $\left(\mathrm{C}_{\text {mic }}\right)$ foi determinada pelo método da irradiação-extração, utilizandose forno de micro-ondas (Islam \& Weil, 1998). O extrator utilizado foi $\mathrm{K}_{2} \mathrm{SO}_{4} 0,5 \mathrm{~mol} \mathrm{~L}^{-1}$ e o carbono contido nos extratos foi quantificado por meio de procedimento por oxidação úmida (Yeomans \& Bremner, 1988). O fator de conversão $\left(\mathrm{K}_{\mathrm{C}}\right)$ usado para converter o fluxo de $\mathrm{C}$ para $\mathrm{C}$ da biomassa microbiana $\left(C_{\text {mic }}\right)$ foi 0,33 (Sparling \& West, 1988). A proporção $C_{\text {mic }} / C O T$ ou quociente microbiano foi calculada, para refletir os aportes de $\mathrm{C}$ e a conversão de substratos orgânicos para o C da biomassa microbiana (Sparling, 1992).

Os estoques de $\mathrm{C}$ e $\mathrm{N}\left(\mathrm{Mg} \mathrm{ha}^{-1}\right)$ no solo foram determinados pela expressão: teor de $\mathrm{C}$ ou $\mathrm{N}\left(\mathrm{kg} \mathrm{Mg}^{-1}\right)$ x Ds x e x 10, em que Ds $=$ densidade do solo $\left(\mathrm{Mg} \mathrm{m}^{-3}\right)$ e e $=$ espessura da camada de solo (m). A densidade do solo nos diferentes sistemas e profundidades foi determinada pelo método do anel volumétrico, conforme EMBRAPA (1997) (Tabela 2).

Tabela 2. Densidade do solo (Ds) em um Latossolo Vermelho-Amarelo sob floresta nativa, plantio convencional e plantio direto com diferentes tempos de implantação no cerrado piauiense

\begin{tabular}{ccccc}
\hline \multirow{2}{*}{ Sistemas $^{\mathbf{1}}$} & \multicolumn{4}{c}{ Densidade do solo $\left(\mathbf{M g ~ m}^{-3}\right)$} \\
\cline { 2 - 5 } & $\mathbf{0 - 5} \mathbf{~ c m}$ & $\mathbf{5 - 1 0} \mathbf{~ c m}$ & $\mathbf{1 0 - 2 0} \mathbf{~ c m}$ & $\mathbf{2 0 - 4 0 ~} \mathbf{~ m}$ \\
FNC & 1,02 & 1,11 & 1,18 & 1,18 \\
PC & 1,19 & 1,16 & 1,27 & 1,38 \\
PD2 & 1,35 & 1,32 & 1,35 & 1,34 \\
PD4 & 1,29 & 1,36 & 1,38 & 1,27 \\
PD6 & 1,28 & 1,39 & 1,35 & 1,26 \\
\hline
\end{tabular}

FNC - Floresta Nativa de Cerrado; PC - Plantio convencional; PD2 - Plantio direto com 2 anos de implantação; PD4 - Plantio direto com 4 anos de implantação; PD6 - Plantio direto com 6 anos de implantação

Avaliou-se o efeito dos diferentes sistemas de manejo nos atributos químicos e biológicos do solo, para cada profundidade, por meio da análise de variância, comparando-se posteriormente as médias, pelo teste de Tukey $(\mathrm{p}<0,05)$ por meio do software SAEG, versão 9.0 .

\section{RESULTADOS E DISCUSSÃO}

Aporte de Ce N pela soja nos diferentes sistemas de preparodo solo

A produtividade de grãos foi maior no sistema $\mathrm{PD}$ em comparação com o PC, com aumento de $17 \%$ para o PD2 e PD4 e de $32 \%$ para o PD6 (Tabela 3 ). Neste estudo, as produtividades de grãos no sistema PD, especialmente no PD6, foram

Tabela 3. Produtividade de grãos e estimativa dos aportes de $\mathrm{C}$ e $\mathrm{N}$ pela cultura da soja sob plantio convencional e plantio direto com diferentes tempos de implantação no cerrado piauiense

\begin{tabular}{|c|c|c|c|c|c|c|c|}
\hline \multirow{3}{*}{ Sistema } & \multirow{3}{*}{$\begin{array}{l}\text { Produtividade } \\
\text { de grãos } \\
\mathrm{kg} \mathrm{ha}^{-1}\end{array}$} & \multicolumn{6}{|c|}{ Aporte $\left(\mathrm{kg} \mathrm{ha}^{-1}\right)$} \\
\hline & & \multicolumn{2}{|c|}{ Raiz } & \multicolumn{2}{|c|}{ Parte aérea } & \multicolumn{2}{|c|}{ Total } \\
\hline & & C & N & C & $\mathbf{N}$ & C & $\mathbf{N}$ \\
\hline $\begin{array}{l}\text { PC } \\
\text { PD2 } \\
\text { PD4 } \\
\text { PD6 }\end{array}$ & $\begin{array}{l}2.733 b^{\#} \\
3.203 a \\
3.200 a \\
3.596 a\end{array}$ & $\begin{array}{l}1.041 \\
1.220 \\
1.219 \\
1.370\end{array}$ & $\begin{array}{l}52 \\
61 \\
61 \\
69\end{array}$ & $\begin{array}{l}2.603 \\
3.051 \\
3.048 \\
3.425\end{array}$ & $\begin{array}{l}130 \\
153 \\
152 \\
171\end{array}$ & $\begin{array}{l}3.644 \mathrm{~b} \\
4.271 \mathrm{a} \\
4.267 \mathrm{a} \\
4.795 \mathrm{a}\end{array}$ & $\begin{array}{l}182 b \\
214 a b \\
213 a b \\
240 a\end{array}$ \\
\hline
\end{tabular}

PC - Plantio convencional; PD2 - Plantio direto com 2 anos de implantação; PD4 - Plantio direto com 4 anos de implantação; PD6 - Plantio direto com 6 anos de implantação; FNC - Floresta Nativa de Cerrado. \# Valores seguidos da mesma letra na coluna não apresentam diferenças significativas entre si pelo teste Tukey a $5 \%$ de probabilidade 
semelhantes àquelas observadas por Secco et al. (2004), que obtiveram, em um Latossolo Vermelho Distroférrico, produtividade de $3.570 \mathrm{~kg} \mathrm{ha}^{-1}$ no sistema plantio direto escarificado, após oito anos consecutivos de uso do sistema.

$\mathrm{O}$ aumento da produtividade no $\mathrm{PD}$ em relação ao PC decorre, provavelmente, da adição regular de palhada, que contribui para a melhoria da estrutura do solo favorecendo a aeração e a infiltração de água permitindo, assim, maior penetração das raízes (Jiao et al., 2006). Por outro lado, o uso contínuo do solo sob sistema PC, possibilita a formação de camadas compactadas, a redução da estabilidade dos agregados e o aparecimento, em maior número, de microporos, aumentando a propensão à perda de solo e aumento à resistência das raízes à penetração (Carvalho et al., 2004).

O uso contínuo da área com PD também aumentou o aporte de $\mathrm{C}$ e $\mathrm{N}$ ao solo quando comparado com o PC. O menor e o maior aporte de $\mathrm{C}$ foram observados no $\mathrm{PC}\left(3.644 \mathrm{~kg} \mathrm{ha}^{-1}\right)$ e no PD6 (4.795 kg ha-1), respectivamente (Tabela 3). O aporte de N foi de 182, 214, 213 e $240 \mathrm{~kg} \mathrm{ha}^{-1}$ nos sistemas PC, PD2, PD4 e PD6, respectivamente, dos quais $28 \%$ fornecidos pelo sistema radicular (Tabela 3). Os maiores aportes de $\mathrm{C}$ e N no PD estão diretamente associados ao aumento da fitomassa aérea e de raízes das culturas de cobertura e comercial adotadas, neste sistema, como consequência das maiores produtividades.

\section{Atributos químicos do solo}

Maiores valores de $\mathrm{pH}(\mathrm{p}<0,05)$ foram verificados no PD na camada $0-5 \mathrm{~cm}$, independente do tempo de adoção, em relação ao PC e FNC; na camada de $5-10 \mathrm{~cm}$, o pH no PD2 foi maior que no $\mathrm{PC}$ e este maior que na FNC. Por outro lado, nas duas últimas camadas os valores de $\mathrm{pH}$, entre os sistemas de preparo, foram similares (Tabela 4). O aumento do $\mathrm{pH}$ nos sistemas cultivados comparativamente aos sistemas naturais, tem sido reportado em diversos estudos (Carneiro et al., 2004; Jantalia et al., 2007) e pode ser atribuído à maior concentração de bases trocáveis na camada superficial do solo, devido à fertilização e correção. Além disto, no caso do PD, comparativamente com o PC, o aumento do pH na camada superficial decorre da aplicação de calcário em superfície sem incorporação (Carvalho et al., 2007; Freiria et al., 2008).

O teor de fósforo extraível (P ext.) foi maior $(\mathrm{p}<0,05)$ no PD especialmente no PD2, em comparação com os outros sistemas, na camada de $0-5 \mathrm{~cm}$ (Tabela 4); no PC, o teor de P ext. foi semelhante nas camadas 0-5, 5-10 e 10-20 cm, provavelmente devido ao revolvimento do solo realizado na camada arável (0$20 \mathrm{~cm}$ ), com subseqüente distribuição uniforme do nutriente. Observou-se também, no sistema PD, variabilidade nos teores de $\mathrm{P}$ no perfil do solo nos diferentes tempos de adoção e entre eles, na mesma camada, com valores maiores na camada superficial (Tabela 4). Este resultado pode estar associado à aplicação localizada de fertilizantes fosfatados, predominantemente nas linhas de semeadura, que se mantêm pouco alteradas com o tempo de cultivo, pelo não revolvimento do solo e pela baixa mobilidade do P (Novais \& Smith, 1999; Schlindwein \& Anghinoni, 2000).

Em todas as camadas, maiores valores de bases trocáveis foram observadas nos sistemas cultivados em relação à FNC. Os teores de $\mathrm{K}$ foram maiores nos sistemas PD4 (0-5 e 5-10 $\mathrm{cm})$, PD6 (5-10 e 10-20 cm) e PC (10-20 cm). Na camada mais profunda $(20-40 \mathrm{~cm})$, os sistemas cultivados não diferiram entre

Tabela 4. A tributos químicos em um Latossolo Vermelho-Amarelo sob floresta nativa, plantio convencional e plantio direto com diferentes tempos de adoção no cerrado piauiense

\begin{tabular}{|c|c|c|c|c|c|c|c|c|c|}
\hline \multirow{2}{*}{ Sistema ${ }^{1}$} & \multirow{2}{*}{ pH } & \multirow{2}{*}{$\begin{array}{c}P^{2} \\
\mathrm{mg} \mathrm{dm^{3 }}\end{array}$} & $\mathrm{K}^{+}$ & $\mathrm{Ca}^{2+}$ & $\mathrm{Mg}^{2+}$ & $\mathrm{Al}^{3+}$ & $\mathrm{H}+\mathrm{Al}$ & $S^{3}$ & CTC $^{4}$ \\
\hline & & & \multicolumn{7}{|c|}{$\mathrm{cmol}_{\mathrm{c}} \mathrm{dm}^{3}$} \\
\hline $\begin{array}{l}\text { FNC } \\
\text { PC } \\
\text { PD2 } \\
\text { PD4 } \\
\text { PD6 } \\
\end{array}$ & $\begin{array}{c}4,17 b^{\#} \\
5,33 b \\
6,09 a \\
5,94 a \\
5,91 a \\
\end{array}$ & $\begin{array}{l}2,11 \text { e } \\
36,2 d \\
80,7 \mathrm{a} \\
61,0 \mathrm{~b} \\
46,3 \mathrm{c}\end{array}$ & $\begin{array}{l}0,03 d \\
0,15 c \\
0,20 b \\
0,59 a \\
0,24 b\end{array}$ & $\begin{array}{l}0,16 \mathrm{c} \\
4,04 \mathrm{~b} \\
6,15 \mathrm{a} \\
4,55 \mathrm{~b} \\
6,27 \mathrm{a} \\
\end{array}$ & $\begin{array}{l}0-5 \mathrm{~cm} \\
0,04 \mathrm{~b} \\
2,13 \mathrm{a} \\
1,89 \mathrm{a} \\
1,58 \mathrm{a} \\
1,72 \mathrm{a}\end{array}$ & $\begin{array}{l}1,55 \mathrm{a} \\
0,18 \mathrm{~b} \\
0,01 \mathrm{c} \\
0,01 \mathrm{c} \\
0,01 \mathrm{c}\end{array}$ & $\begin{array}{c}11,1 \mathrm{a} \\
6,73 \mathrm{~b} \\
3,58 \mathrm{c} \\
5,40 \mathrm{bc} \\
5,67 \mathrm{~b} \\
\end{array}$ & $\begin{array}{l}0,23 \mathrm{c} \\
6,32 \mathrm{~b} \\
8,25 \mathrm{a} \\
6,71 \mathrm{~b} \\
8,23 \mathrm{a}\end{array}$ & $\begin{array}{c}12,9 a b \\
13,2 a b \\
11,8 b \\
12,1 a b \\
13,9 a\end{array}$ \\
\hline $\begin{array}{l}\text { FNC } \\
\text { PC } \\
\text { PD2 } \\
\text { PD4 } \\
\text { PD6 }\end{array}$ & $\begin{array}{c}4,34 c \\
5,39 b \\
5,96 a \\
5,54 a b \\
5,54 a b\end{array}$ & $\begin{array}{c}2,03 d \\
36,5 a \\
30,4 b c \\
29,0 c \\
33,6 a b\end{array}$ & $\begin{array}{c}0,02 \mathrm{c} \\
0,14 \mathrm{~b} \\
0,15 \mathrm{~b} \\
0,20 \mathrm{ab} \\
0,22 \mathrm{a}\end{array}$ & $\begin{array}{l}0,03 \mathrm{c} \\
3,36 \mathrm{~b} \\
5,67 \mathrm{a} \\
3,00 \mathrm{~b} \\
4,66 \mathrm{a}\end{array}$ & $\begin{array}{c}5-10 \mathrm{~cm} \\
0,03 \mathrm{c} \\
1,65 \mathrm{a} \\
1,27 \mathrm{ab} \\
0,81 \mathrm{~b} \\
0,80 \mathrm{~b}\end{array}$ & $\begin{array}{l}1,25 a \\
0,15 b \\
0,02 c \\
0,02 c \\
0,07 c\end{array}$ & $\begin{array}{c}9,16 a \\
6,69 \mathrm{bc} \\
5,06 \mathrm{c} \\
8,33 \mathrm{ab} \\
7,92 \mathrm{ab}\end{array}$ & $\begin{array}{r}0,08 c \\
5,15 b \\
7,09 a \\
4,02 b \\
5,68 a b\end{array}$ & $\begin{array}{c}10,5 \mathrm{~b} \\
12,0 \mathrm{ab} \\
12,2 \mathrm{ab} \\
12,6 \mathrm{a} \\
13,7 \mathrm{a}\end{array}$ \\
\hline $\begin{array}{l}\text { FNC } \\
\text { PC } \\
\text { PD2 } \\
\text { PD4 } \\
\text { PD6 }\end{array}$ & $\begin{array}{c}4,74 b \\
5,24 a b \\
5,51 a \\
4,84 a b \\
5,44 a b\end{array}$ & $\begin{array}{l}1,96 c \\
32,7 a \\
5,79 b \\
7,94 b \\
5,55 b\end{array}$ & $\begin{array}{l}0,02 c \\
0,14 a \\
0,09 b \\
0,10 b \\
0,13 a\end{array}$ & $\begin{array}{l}0,04 \mathrm{~d} \\
2,68 \mathrm{~b} \\
3,83 \mathrm{a} \\
1,23 \mathrm{c} \\
1,65 \mathrm{c}\end{array}$ & $\begin{array}{c}10-20 \mathrm{~cm} \\
0,03 \mathrm{c} \\
1,21 \mathrm{a} \\
0,57 \mathrm{~b} \\
0,51 \mathrm{~b} \\
0,39 \mathrm{~b}\end{array}$ & $\begin{array}{l}1,37 a \\
0,30 b \\
0,21 b \\
0,98 a \\
0,34 b\end{array}$ & $\begin{array}{c}8,50 a b \\
6,99 b c \\
5,70 c \\
10,3 a \\
7,10 b c\end{array}$ & $\begin{array}{l}0,09 \mathrm{c} \\
4,03 \mathrm{a} \\
4,49 \mathrm{a} \\
1,85 \mathrm{~b} \\
2,17 \mathrm{~b}\end{array}$ & $\begin{array}{c}9,96 \mathrm{~b} \\
11,3 \mathrm{ab} \\
10,4 \mathrm{ab} \\
13,1 \mathrm{a} \\
9,61 \mathrm{~b}\end{array}$ \\
\hline $\begin{array}{l}\text { FNC } \\
\text { PC } \\
\text { PD2 } \\
\text { PD4 } \\
\text { PD6 }\end{array}$ & $\begin{array}{l}5,04 a \\
4,48 a \\
5,09 a \\
4,63 a \\
4,96 a\end{array}$ & $\begin{array}{l}1,53 b \\
3,42 a \\
2,53 a b \\
1,98 a b \\
2,44 a b\end{array}$ & $\begin{array}{l}0,01 \mathrm{~b} \\
0,06 \mathrm{a} \\
0,07 \mathrm{a} \\
0,06 \mathrm{a} \\
0,05 \mathrm{a}\end{array}$ & $\begin{array}{l}0,03 c \\
0,28 b \\
0,68 a \\
0,24 b \\
0,69 a\end{array}$ & $\begin{array}{c}20-40 \mathrm{~cm} \\
0,03 \mathrm{~b} \\
0,21 \mathrm{a} \\
0,21 \mathrm{a} \\
0,22 \mathrm{a} \\
0,20 \mathrm{a}\end{array}$ & $\begin{array}{c}0,99 a \\
0,84 a b \\
0,65 b \\
1,01 a \\
0,71 b\end{array}$ & $\begin{array}{l}6,27 a \\
6,23 a \\
5,23 b \\
6,40 a \\
6,02 a b\end{array}$ & $\begin{array}{l}0,07 c \\
0,56 b \\
0,96 a \\
0,52 b \\
1,13 a\end{array}$ & $\begin{array}{l}7,34 \mathrm{a} \\
7,62 \mathrm{a} \\
6,84 \mathrm{a} \\
7,93 \mathrm{a} \\
7,87 \mathrm{a}\end{array}$ \\
\hline
\end{tabular}


si (Tabela 4). Houve decréscimo dos teores de K no perfil do solo no PD, independente do tempo de adoção, diferente do observado no PC, que apresentou teores semelhantes nas camadas 0-5, 5-10 e 10-20 cm. A aplicação de fertilizantes e corretivos no sistema PD pode causar acúmulo de nutrientes na camada superficial do solo uma vez que, neste sistema, há redução no preparo do solo com menor revolvimento. Isto evita que os nutrientes sejam distribuídos na camada arável do solo e lixiviados (Ciotta et al., 2004), como ocorre com as bases trocáveis, principalmente $\mathrm{K}^{+}$, em solos sob $\mathrm{PC}$ em regiões com elevada pluviosidade. A lixiviação das bases trocáveis ocorre devido à combinação de diversos fatores, dentre eles a precipitação pluviométrica, o manejo do solo, o tipo e a forma de adubação.

Maiores teores de Ca foram observados no PD6 e PD2, intermediários no PD4 e PC e menores na FNC nas camadas 05, 5-10 e 20-40 cm, e se concentraram na camada $0-20 \mathrm{~cm}$, diminuindo aproximadamente $50 \%$ da camada $10-20$ para a de $20-40 \mathrm{~cm}$. Com relação ao Mg não houve, na camada de $0-5 \mathrm{~cm}$, diferenças entre os sistemas cultivados; por outro lado, nas camadas seguintes $(5-10$ e 10-20 cm), o PC apresentou os maiores teores, comparativamente com o PD e FNC (Tabela 4). Esses resultados ratificam a maior concentração de bases trocáveis na camada arável do solo, mais especificamente, na camada 0-20 cm para o PC e na de 0-10 cm para o PD, cujos resultados realçam o efeito da aplicação de fertilizantes e corretivos na superfície do solo no sistema PD, uma vez que são realizadas sobre a superfície, sem incorporação, diminuindo a superfície de contato entre as partículas de solo e as do corretivo retardando, desta forma, os efeitos da calagem e restringindo as reações aos centímetros superficiais do solo (Ciotta et al., 2004; Freiria et al., 2008). Resultados semelhantes foram reportados por Carvalho et al. (2007), que avaliaram mudanças nos atributos químicos do solo sob PD e PC, com diferentes tempos de adoção, na região do Cerrado amazonense, detectando acumulação de $\mathrm{P}$ extraível e bases trocáveis nas camadas 0-5 e 5-10 cm no PD e distribuição mais uniforme até $30 \mathrm{~cm}$ no solo sob PC.

Os teores de $\mathrm{Al}$ e a acidez potencial $(\mathrm{H}+\mathrm{Al})$ foram menores no PD, quando comparados com a FNC e o PC o que, provavelmente, está relacionado à aplicação superficial do calcário sem incorporação, havendo acúmulo de cálcio e magnésio trocáveis e elevação do $\mathrm{pH}$ na superfície do solo, além de maiores teores de matéria orgânica, que aumentam a quantidade de sítios de adsorção catiônicos no solo complexando o Al (Bayer et al., 2004; Almeida et al., 2005).

\section{Teores e estoques de carbono orgânico total e microbiano do solo}

Nas camadas de 0-5 e 5-10 cm, os teores de COT foram maiores ( $\mathrm{p}<0,05)$ no PD, quando comparados com a FNC e ao PC (Tabela 5); entretanto, entre os tempos de adoção, na camada 0-5 cm, o COT foi maior no PD6 que no PD2 e o PD4 não diferiu desses. Na camada 10-20 cm, o teor de COT apresentou o gradiente PD6 > PD4 > PC > FNC, com o sistema PD2 não diferindo dos sistemas PD4 e PC (Tabela 5).

A presença de maiores teores de COT no sistema PD, até 20 $\mathrm{cm}$, está relacionada ao maior aporte de fitomassa na superfície e a redução no uso de implementos agrícolas para o preparo do
Tabela 5. Teor médio de carbono orgânico total (COT), carbono microbiano $\left(C_{\text {mic }}\right)$ e relação $C_{\text {mic }} /$ COT em um Latossolo Vermelho A marelo sob floresta nativa, plantio convencional e plantio direto com diferentes tempos de implantação no cerrado piauiense

\begin{tabular}{cccc}
\hline \multirow{2}{*}{ Sistema } & COT & $\mathbf{C}_{\text {mic }}$ & \multicolumn{1}{c}{$\mathbf{C}_{\text {mic }} / \mathbf{C O T}$} \\
\cline { 2 - 3 } & \multicolumn{3}{c}{$\mathbf{g ~ k g}^{-1}$} \\
FNC & $22,6 \mathrm{c}^{\#}$ & $0-5 \mathrm{~cm}$ & \\
PC & $19,1 \mathrm{c}$ & $0,309 \mathrm{~b}$ & $1,37 \mathrm{a}$ \\
PD2 & $25,2 \mathrm{~b}$ & $0,361 \mathrm{ab}$ & $0,91 \mathrm{~b}$ \\
PD4 & $28,0 \mathrm{ab}$ & $0,341 \mathrm{ab}$ & $1,24 \mathrm{a}$ \\
PD6 & $29,5 \mathrm{a}$ & $0,403 \mathrm{a}$ & $1,37 \mathrm{a}$ \\
\hline & & $5-10 \mathrm{~cm}$ & \\
FNC & $16,4 \mathrm{~b}$ & $0,278 \mathrm{a}$ & $1,70 \mathrm{a}$ \\
PC & $18,1 \mathrm{~b}$ & $0,158 \mathrm{~b}$ & $0,89 \mathrm{c}$ \\
PD2 & $21,7 \mathrm{a}$ & $0,265 \mathrm{a}$ & $1,23 \mathrm{~b}$ \\
PD4 & $24,2 \mathrm{a}$ & $0,272 \mathrm{a}$ & $1,12 \mathrm{bc}$ \\
PD6 & $24,4 \mathrm{a}$ & $0,276 \mathrm{a}$ & $1,13 \mathrm{bc}$ \\
\hline & & $10-20 \mathrm{~cm}$ & \\
FNC & $13,2 \mathrm{~d}$ & $0,184 \mathrm{a}$ & $1,40 \mathrm{a}$ \\
PC & $15,0 \mathrm{c}$ & $0,138 \mathrm{~b}$ & $0,92 \mathrm{~b}$ \\
PD2 & $15,9 \mathrm{bc}$ & $0,213 \mathrm{a}$ & $1,34 \mathrm{a}$ \\
PD4 & $17,1 \mathrm{~b}$ & $0,200 \mathrm{a}$ & $1,17 \mathrm{ab}$ \\
PD6 & $21,9 \mathrm{a}$ & $0,204 \mathrm{a}$ & $0,93 \mathrm{~b}$ \\
\hline & & $20-40 \mathrm{~cm}$ & \\
FNC & $11,1 \mathrm{bc}$ & $0,169 \mathrm{a}$ & $1,52 \mathrm{a}$ \\
PC & $13,8 \mathrm{a}$ & $0,134 \mathrm{~b}$ & $0,97 \mathrm{c}$ \\
PD2 & $9,65 \mathrm{c}$ & $0,131 \mathrm{~b}$ & $1,40 \mathrm{ab}$ \\
PD4 & $12,6 \mathrm{ab}$ & $0,131 \mathrm{~b}$ & $1,04 \mathrm{bc}$ \\
PD6 & $11,9 \mathrm{ab}$ & $0,136 \mathrm{~b}$ & $1,15 \mathrm{bc}$ \\
\hline
\end{tabular}

FNC - Floresta Nativa de Cerrado; PC - Plantio convencional; PD2 - Plantio direto com 2 anos de implantação; PD4 - Plantio direto com 4 anos de implantação; PD6 - Plantio direto com 6 anos de implantação. \# Valores seguidos da mesma letra na coluna não apresentam diferenças significativas entre si pelo teste Tukey a $5 \%$ de probabilidade

solo, aumentando a quantidade de MO e diminuindo a decomposição do material orgânico protegido fisicamente nos agregados do solo (Jantalia et al., 2007).

Em áreas sob PC, geralmente o teor de COT é menor na camada superficial, maior, em profundidade e mais uniforme no perfil do solo, quando comparado com o PD; isto ocorre porque, provavelmente, nos sistemas em que o solo é revolvido a MO é distribuída por toda a camada arável, fazendo com que os teores de COT, em profundidades maiores, possam ser semelhantes ou até maiores que no sistema PD (Jantalia et al., 2007; Baker et al., 2007; Ussiri \& Lal, 2009).

Os teores de $\mathrm{C}_{\text {mic. }}$ foram maiores no PD6, intermediários na FNC e menores no PC na camada $0-5 \mathrm{~cm}$, considerando-se que os sistemas PD4 e PD2 não diferiram do PD6 e da FNC (Tabela 5). Resultados semelhantes foram obtidos por Leite et al. (2007) que observaram menores valores do $\mathrm{C}_{\text {mic }}$ em sistemas cultivados quando comparados aos da floresta nativa na camada superficial $(0-5 \mathrm{~cm})$.

Nas camadas 5-10 e 10-20 cm, o PC foi o sistema que apresentou os menores teores de $\mathrm{C}_{\text {mic }}$ em comparação com os demais sistemas, que não diferiram entre si. A presença de maiores teores de $\mathrm{C}_{\text {mic }}$ no $\mathrm{PD}$ e $\mathrm{FNC}$ nos primeiros $20 \mathrm{~cm}$, pode ser atribuída ao aumento da MO, que irá proporcionar incremento na atividade e na quantidade da biomassa microbiana, uma vez que o $C$ é fonte de energia e nutrientes para os micro-organismos (Brussaard et al., 2007; Colodro et al., 2007). 
A implantação do sistema PD após dois anos proporcionou aumento de $12 \%$ no COT e de $17 \%$ no $\mathrm{C}_{\text {mic }}$ e, depois de seis anos, de $30 \%$ para ambos, na camada $0-5 \mathrm{~cm}$ em relação à FNC. $\mathrm{O}$ aumento no sistema $\mathrm{PD} 2$ pode ser resultante da rápida transição (3 anos) do PC para o PD, diminuindo o tempo de perturbação do solo. Os maiores teores de COT e $\mathrm{C}_{\text {mic }}$ no PD e menores no PC podem na maioria dos casos e em comparação com a FNC, indicar que os solos sob esses sistemas têm atuado como dreno (PD) e fonte (PC) de C para a atmosfera. Isto representa importante contribuição da agricultura conservacionista para atenuar a concentração de $\mathrm{CO}_{2}$ na atmosfera e nas alterações climáticas globais (Six et al., 2004; Zinn et al., 2005).

O quociente microbiano na camada $0-5 \mathrm{~cm}$ foi maior $(\mathrm{p}<$ 0,05) na FNC e no PD2 e PD6 e menor no PC, considerando-se que o PD4 não diferiu de nenhum dos sistemas (Tabela 5). Na camada 5-10 cm, o quociente microbiano foi maior na FNC, intermediário no PD2 e menor no PC, com os sistemas PD4 e PD6 não diferindo do PD2 e PC. Na camada seguinte, o maior valor foi observado na FNC e no PD2 e o menor no PD6 e PC, com PD4 não diferindo de nenhum dos sistemas. Por fim, na camada de 20-40 cm, a FNC continuou com o maior valor e o PC com o menor, com o PD2 se diferenciando apenas do PC e o PD4 e PD6, da FNC. A presença de maior quociente microbiano na floresta está associada, provavelmente, ao equilíbrio existente no sistema por conta da estreita e harmônica integração da cobertura vegetal com o sistema físico, químico e biológico do solo, por meio dos processos essenciais, como a ciclagem de nutrientes, resultante da formação e decomposição da matéria orgânica (Leite et al., 2003). Os menores valores observados no sistema PC implicam em menor disponibilidade de COT para a microbiota do solo. Este resultado pode ser observado a médio e longo prazos após a adoção do PC, pois o preparo do solo quebra os seus agregados, disponibilizando substrato orgânico para a biomassa microbiana do solo, aumentando a taxa de decomposição e da comunidade microbiana e reduzindo os teores de matéria orgânica do solo (Ferreira et al., 2007).

O estoque de COT na camada $0-20 \mathrm{~cm}$ foi maior $(\mathrm{p}<0,05)$ no PD6, intermediário no PD2, com o PD4 não diferindo desses, e menor no PC e na FNC; quando se estimou o estoque para a camada 0-40 cm, o comportamento foi semelhante; contudo, a FNC apresentou o menor estoque, diferindo de todos os outros sistemas (Figura 1A). Maiores valores de COT no PC em relação à $\mathrm{FNC}$, na camada $0-40 \mathrm{~cm}$, podem ser atribuídos, além da baixa produção de biomassa das florestas de Cerrado do Meio-Norte brasileiro (Azevedo et al., 2007), à incorporação dos restos vegetais da cultura principal deixada sobre o solo, possibilitando a distribuição da matéria orgânica por toda a camada arável e permitindo que os teores de carbono orgânico (CO), em profundidades maiores, possam ser semelhantes ou até maiores que nos sistemas conservacionistas (Ferreira et al., 2007).

O estoque do $\mathrm{C}_{\text {mic }}$, nas camadas $0-20$ e $0-40 \mathrm{~cm}$ foi maior no $\mathrm{PD}$, independente do tempo de adoção, intermediário na FNC e menor no PC (Figura 1B). O menor estoque de $\mathrm{C}_{\text {mic }}$ no sistema PC em comparação ao PD se deve, provavelmente, ao maior revolvimento do solo por meio das operações de preparo causando desequilíbrio no sistema e, em consequência, a aceleração do processo de decomposição da matéria orgânica que em longo prazo, irá diminuir o teor de matéria orgânica do solo e da biomassa microbiana (Leite et al., 2003; Brussaard et al., 2007).

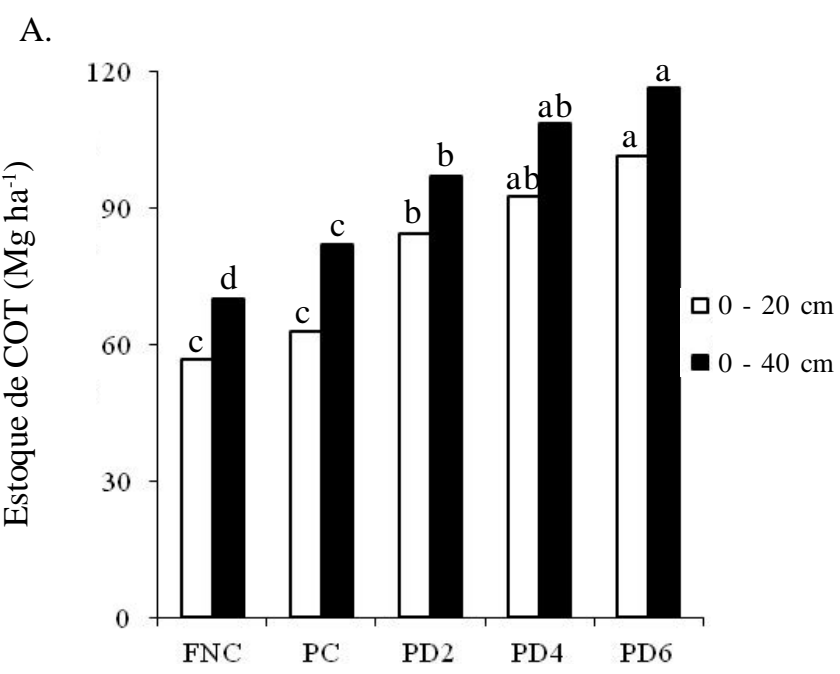

B.

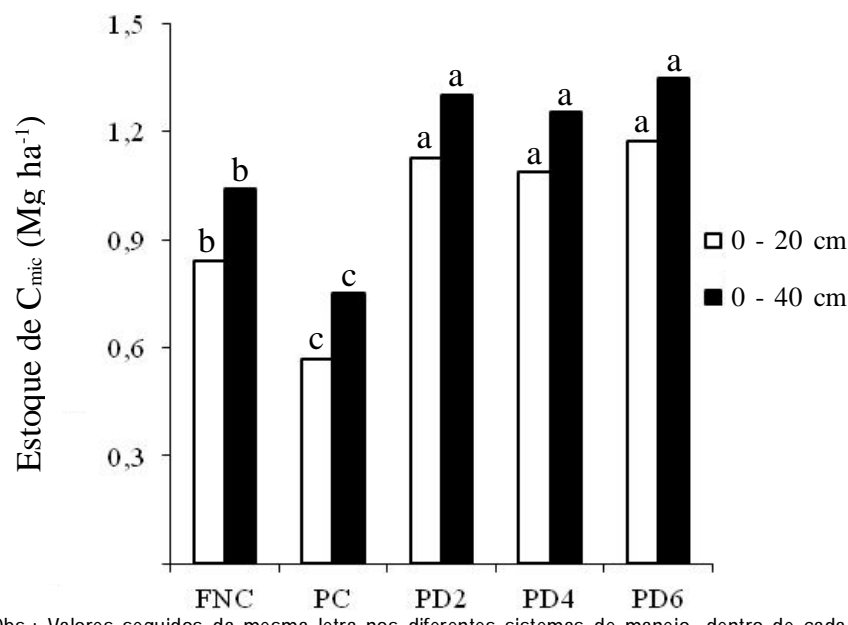

Obs.: Valores seguidos da mesma letra nos diferentes sistemas de manejo, dentro de cada profundidade, não apresentam diferenças significativas entre si pelo teste Tukey a $5 \%$ de probabilidade Figura 1. Estoque de carbono orgânico total (COT) (A) e estoque de carbono da biomassa microbiana $\left(C_{\text {mic }}\right)(B)$ nas camadas 0-20 cm e 20-40 cm em Latossolo VermelhoAmarelo sob floresta nativa de cerrado (FNC), plantio convencional (PC) e plantio direto (PD2), 4 anos (PD 4) e 6 anos (PD 6) de implantação no cerrado piauiense

Os maiores estoques de COT e $\mathrm{C}_{\text {mic }}$ estão localizados na camada 0-20 cm, independente do sistema de cultivo, com percentuais acima de $75 \%$ para o COT e $\mathrm{C}_{\text {mic }}$ do estoque total da camada 0-40 cm (Figura 1A, 1B). Azevedo et al. (2007) verificaram, na camada de $0-5 \mathrm{~cm}$, maior estoque de COT em solo sob PD (7,72 $\left.\mathrm{Mg} \mathrm{ha}^{-1}\right)$, comparativamente àqueles sob preparo convencional (com grades) $\left(5,58 \mathrm{Mg} \mathrm{ha}^{-1}\right)$ e preparo reduzido (com escarificador) $\left(7,19 \mathrm{Mg} \mathrm{ha}^{-1}\right)$. Esses resultados estão de acordo com os observados na maioria dos estudos, que preconizam maiores estoques do COT sob PD em comparação com os sistemas convencionais e que este acúmulo 
é restrito às camadas superficiais (Bayer et al., 2006; Xiao-Bin et al., 2006).

\section{ConClusões}

1. A implantação do sistema plantio direto melhora os atributos químicos e aumenta o estoque de carbono orgânico total e microbiano do solo, até $20 \mathrm{~cm}$ de profundidade.

2. O sistema plantio direto é uma alternativa sustentável para a melhoria da qualidade do solo, em áreas do cerrado piauiense.

\section{Agradecimentos}

Ao CNPq, pelo auxílio financeiro.

\section{LITERATURA CITADA}

Aguiar, T. J. A.; Monteiro, M. S. L. Modelo agrícola e desenvolvimento sustentável: A ocupação do cerrado piauiense. Ambiente e Sociedade, v.8, n.2, p.1-18, 2005.

Al-Kaisi, M. M.; Yin, X. H. Tillage and crop residue effects on soil carbon and carbon dioxide emission in corn-soybean rotations. Journal of Environmental Quality, v.34, n.2, p.437445. 2005.

Almeida, J. A.; Bertol, I.; Leite, D.; Amaral, A. J.; Zoldan Júnior, W. A. Propriedades químicas de um Cambissolo Húmico sob preparo convencional e semeadura direta após seis anos de cultivo. Revista Brasileira de Ciência do Solo, v.29, n.3, p.437-445, 2005.

Azevedo, D. M. P.; Leite, L. F. C.; Teixeira Neto, M. L.; Dantas, J. S. Atributos físicos e químicos de um Latossolo Amarelo e distribuição do sistema radicular da soja sob diferentes sistemas de preparo no cerrado maranhense. Revista Ciência Agronômica, v.38, n.1, p.32-40, 2007.

Baker, J. M.; Ochsner, T. E.; Venterea, R. T.; Griffis, T. J. Tillage and soil carbon sequestration-what do we really know? Agriculture Ecosystem \& Enviroment, v.118, n.1, p.1-5, 2007.

Bayer, C.; Martin-Neto, L.; Mielniczuk, J.; Pavinato, A. Armazenamento de carbono em frações lábeis da matéria orgânica de um Latossolo Vermelho sob plantio direto. Pesquisa Agropecuária Brasileira, v.39, n.7, p.677-683, 2004.

Bayer, C.; Martin-Neto, L.; Mielniczuk, J.; Pavinato, A.; Dieckow, J. Carbon sequestration in two Brazilian Cerrado soils under no-till. Soil \& Tillage Research, v.86, n.2, p.237245, 2006.

Brussaard, L.; Ruiter, P. C.; Brown, G. G. Soil biodiversity for agricultural sustainability. Agriculture Ecosystems \& Environment, v.121, n.3, p.233-244, 2007.

Carneiro, R. G.; Mendes, I. C.; Lovato, P. E.; Carvalho, A. M.; Vivaldi, L. J. Indicadores biológicos associados ao ciclo do fósforo em solos de Cerrado sob plantio direto e plantio convencional. Pesquisa Agropecuária Brasileira, v.39, n.7, p.661-669, 2004.
Carvalho, J. L. N.; Cerri, C. E. P.; Feigl, B. J.; Píccolo, M. C.; Godinho, V. P.; Herpin, U. Changes of chemical properties in na oxisol after clearing of native Cerrado vegetation for agricultural use in Vilhena, Rondônia State, Brazil. Soil \& Tillage Research, v.96, n.1, p.95-102, 2007.

Carvalho, M. A. C.; Athayde, M. L. F.; Soratto, R. P.; Alves, M. C.; Arf, O. Soja em sucessão a adubos verdes no sistema plantio direto e convencional em solo de Cerrado. Pesquisa Agropecuária Brasileira, v.39, n.11, p.1141-1148, 2004.

Ciotta, M. N.; Bayer, C.; Ernani, P. R.; Fontoura, S. M. V.; Wobeto, C.; Albuquerque, J. A. Manejo da calagem e os componentes da acidez de Latossolo Bruno em plantio direto. Revista Brasileira de Ciência do Solo, v.28, n.1, p.317326, 2004.

Colodro, G.; Espíndola, C. R.; Cassiolato, A. M. R; Alves, M. C. Atividade microbiana em um Latossolo degradado tratado com lodo de esgoto. Revista Brasileira de Engenharia Agrícola e Ambiental, v.11, n.2, p.195-198, 2007.

CPRM - Companhia de Pesquisa de Recursos Minerais. Projeto cadastro de fontes de abastecimento por água subterrânea, estado do Piauí: diagnóstico do município de Baixa Grande do Ribeiro. Aguiar, R. B.; Gomes, J. R. C. Fortaleza: CPRM, Serviço Geológico do Brasil, 2004.

EMBRAPA - Empresa Brasileira de Pesquisa Agropecuária. Manual de métodos de análises de solos. 2.ed. Rio de Janeiro: Embrapa Solos, 1997. 212p.

Ferreira, E. A. B.; Resck, D. V. S.; Gomes, A. C.; Ramos, M. L. G. Dinâmica do carbono da biomassa microbiana em cinco épocas do ano em diferentes sistemas de manejo do solo no cerrado. Revista Brasileira de Ciência do Solo, v.31, n.6, p.1625-1635, 2007.

Fonseca, G. C.; Carneiro, M. A. C.; Costa, A. R. da; Oliveira, G. C.; Balbino, L. C. Atributos físicos, químicos e biológicos de um Latossolo Vermelho Distrófico de cerrado sob duas rotações de cultura. Pesquisa Agropecuária Tropical, v.37, n.1, p.22-30, 2007.

Freiria, A. C.; Mantovani, J. R.; Ferreira, M. E.; Cruz, M. C. P.; Yagi, R. Alterações em atributos químicos do solo pela aplicação de calcário na superfície ou incorporado. Acta Scientiarum Agronomy, v.30, n.2, p.285-291, 2008.

Islam, K. R.; Weil, R. R. Microwave irradiation of soil for routine measurement of microbial biomass carbon. Biology and Fertility of Soil, v.27, n.4, p.408-416, 1998.

Jantalia, C. P.; Resck, D. V. S.; Alves, B. J. R.; Zotarelli, L.; Urquiaga, S.; Boddey, R. M. Tillage effect on C stocks of a clayey Oxisol under a soybean-based crop rotation in the Brazilian Cerrado region. Soil \& Tillage Research, v.95, n.1, p.97-109, 2007.

Jiao, Y.; Whalen, J, K.; Hendershot, W. H. No-tillage and manure applications increase aggregation and improve nutrient retention in a sandy-loam soil. Geoderma, v.134, n.1 , p.2433, 2006.

Kliemann, H. J.; Braz, A. J. P. B. Taxas de decomposição de resíduos de espécies de cobertura em Latossolo Vermelho distroférrico. Pesquisa Agropecuária Tropical, v.36, n.1, p.2128, 2006. 
Leite, L. F. C.; Mendonça, E. S.; Machado, P. L. O. A. Influence of organic and mineral fertilisation on organic matter fractions of a Brazilian Acrisol under maize/common bean intercrop. Australian Journal of Soil Research, v.45, n.1, p.25-32, 2007.

Leite, L. F. C; Mendonça, E. S.; Machado, P. L. O. A.; Matos, E. $\mathrm{S}$. Total $\mathrm{C}$ and $\mathrm{N}$ storage and organic $\mathrm{C}$ pools of a RedYellow Podzolic under conventional and no tillage at the Atlantic Forest Zone, Southeastern Brazil. Australian Journal of Soil Research, v.41, n.4, p.717-730, 2003.

Lestari, P.; Van, K.; Lee, S. Nodulation and growth of a supernodulating soybean mutant SS2-2 symbiotically associated with Bradyrhizobium japonicum. Journal AgroBiogen, v.2, n.1, p.8-15, 2006.

Mallarino, A. P.; Borges, R. Phosphorus and potassium distribution in soil following long-term deep-band fertilization in different tillage systems. Soil Science Society of America Journal, v.70, n.2, p.702-707, 2006.

Murage, E. W.; Voroney, P. R.; Kay, B. D.; Deen, B.; Beyaert, R. P. Dynamics and turnover of soil organic matter as affected by tillage. Soil Science Society of America Journal, v.71, n.4, p.1363-1370, 2007.

Novais, R. F.; Smith, T. J. Fósforo em solo e planta em condições tropicais. Viçosa: UFV-DPS. 1999. 399p.

Schlindwein, J. A.; Anghinoni, I. Variabilidade vertical de fósforo e potássio disponíveis e profundidade de amostragem do solo no sistema plantio direto. Ciência Rural, v.30, n.4, p.611617,2000

Secco, D.; Reinert, D. J.; Reichert, J. M.; Ros, C. O. da. Produtividade de soja e propriedades físicas de um Latossolo submetido a sistemas de manejo e compactação. Revista Brasileira de Ciência do Solo, v.28, n.5, p.797-804, 2004.

Silva, V. R.; Reinert, D. J.; Reichert, J. M. Densidade do solo, atributos químicos e sistema radicular do milho afetados pelo pastejo e manejo do solo. Revista Brasileira de Ciência do Solo, v.24, n.1, p.191-199, 2000.

Silveira, P. M.; Stone, L. F. Teores de nutrientes e matéria orgânica afetados pela rotação de culturas e sistema de preparo do solo. Revista Brasileira de Ciência do Solo, v.25, n.2, p.387-394, 2001.
Six, J.; Ogle, S. M.; Breidt, F. J.; Conant, R. T.; Mosier, A. R.; Paustian, K. Potential to mitigate global warming with no-tillage management is only realized when practiced in the long term. Global Change Biology, v.10, n.1, p.155$160,2004$.

Souza, Z. M.; Alves, M. C. Propriedades químicas de um Latossolo Vermelho distroférrico de cerrado sob diferentes usos e manejos. Revista Brasileira de Ciência do Solo, v.27, n.1, p.133-139, 2003.

Sparling, G. P. Ratio of microbial biomass carbon to soil organic carbon as a sensitive indicator of changes in soil organic matter. Australian Journal of Soil Research, v.30, n.2, p.195207, 1992.

Sparling, G. P.; West, A. W. Adirect extraction method to estimate soil microbial C: Calibration in situ using microbial respiration and ${ }^{14} \mathrm{C}$ labelled cells. Soil Biology and Biochemistry, v.20, n.3, p.337-343, 1988.

Thomas, G. A.; Dalal, R. C.; Standley, J. No-till effects on organic matter, $\mathrm{pH}$, cation exchange capacity and nutrient distribution in a Luvisol in the semi-arid subtropics. Soil \& Tillage Research, v.94, n.2, p.295-304, 2007.

Ussiri, D. A. N.; Lal, R. Long-term tillage effects on soil carbon storage and carbon dioxide emissions in continuous corn cropping system from an alfisol in Ohio. Soil \& Tillage Research, v.104, n.1, p.39-47, 2009.

Xiao-Bin, W.; Dian-Xiong, C.; Hoogmoed, W. B.; Oenema, O.; Perdok, U. D. Potential effect of conservation tillage on sustainable land use: a review of global long-term studies. Pedosphere, v.16, n.5, p.587-595, 2006.

Yeomans, J. C.; Bremner, J. M. A rapid and precise method for routine determination of organic carbon in soil. Communications in Soil Science and Plant Analysis, v.19, n.13, p.1467-1476, 1988.

Zinn, Y. L.; Lal, R.; Resck, D. V. S. Changes in soil organic carbon stocks under agriculture in Brazil. Soil \& Tillage Research, v.84, n.1, p.28-40, 2005. 